\title{
Echocardiographic Abnormalities and Antiphospholipid Antibodies in Patients with Systemic Lupus Erythematosus
}

\author{
Creso Abreu Falcão, Izabel Cristina Alves, Wiliam Habib Chahade, \\ Ângela Luzia Branco Pinto Duarte, Norma Lucena-Silva
}

Recife, PE - São Paulo, SP - Brazil

\begin{abstract}
Objective - Lupus anticoagulant and anticardiolipin antibodies $(a C L)$ have been associated with thrombosis, recurrent abortion, and thrombocytopenia in patients with systemic lupus erythematosus (SLE), but their relationship with cardiac disease is less clear. The purpose of this study was to evaluate the association between antiphospholipid antibodies (aPL) and echocardiographic abnormalities in patients with SLE.
\end{abstract}

Methods - A total of 70 consecutive patients and 42 control subjects underwent M-mode, 2-dimensional and Doppler echocardiography and tests for lupus anticoagulant, aCL IgG, IgM, and IgA. Lupus anticoagulant was assayed with the dilute Russell viper venom time, and $a C L$ $\operatorname{Ig} G$, IgM, and $\operatorname{Ig} A$ were measured by an enzyme-linked immunosorbent assay (ELISA).

Results - Lupus anticoagulant showed a prevalence of $10 \%$. As a whole, aCL had a prevalence of $44.3 \%$ and aPL had a prevalence of $50 \%$. Patients with echocardiographic abnormalities had a prevalence of $54.3 \%$ and showed a trend towards an association with aCL $\operatorname{IgG}$ $(P=0.06)$. The presence of pulmonary hypertension $(\mathrm{PH})$ was significantly associated with aCL $\operatorname{Ig} G(p=0.02)$.

Conclusion - aCL IgG was significantly associated with PH and showed a strong trend towards an association with echocardiographic abnormalities taken together. These findings suggest a role for aCL IgG in the development of lupus cardiovascular disease.

Keywords: lupus erythematosus, antiphospholipid syndrome, lupus coagulation inhibitor

Universidade de Pernambuco-FESP, Hospital dos Servidores do Estado de Pernambuco, Hospital do Servidor Público Estadual de São Paulo, Universidade Federal de Pernambuco e Centro de Pesquisas Aggeu Magalhães/Fundação Oswaldo Cruz Mailing address: Creso Abreu Falcão, Rua Padre Carapuceiro, 488/603 - 51020-280 Recife, PE - e-mail: creso@hoelink.com.br

Received to publication on $5 / 22 / 01$

Accepted on 12/11/01
A number of relevant studies in patients with systemic lupus erythematosus (SLE) have evidenced an association of antiphospholipid antibodies (aPL) with cardiopathy, particularly in the case of Libman-Sacks endocarditis, valvar dysfunction, and valvar thickening ${ }^{1-5}$. Specific associations of aPL with pulmonary hypertension $(\mathrm{PH})^{6}$, myocardial dysfunction ${ }^{3}$, and ischemic cardiopathy ${ }^{7,8}$ in lupus patients have also been reported. These findings suggest that aPLs are responsible for basic immunological events related to the development of cardiopathy in SLE- and that some cardiac abnormalities may be considered as real components of primary or secondary aPL syndrome (APS) ${ }^{9}$. However, other authors were not able to demonstrate an association between cardiac disease in SLE and aPL ${ }^{10-12}$.

The similarity between valvar lesions detected in patients with primary aPL syndrome and valvar lesions of lupus patients has reinforced the statistical associations observed ${ }^{9}$. Also, the detection of selective deposition of immunoglobulins and complement at valve vegetations of Libman-Sacks endocarditis ${ }^{9}$, as well as the more recent findings concerning the deposition of IgG isotype ACL in the subendothelial layer of heart valves in patients with APS ${ }^{13}$, have consistently suggested a role for immunological disturbances in the development of valvar lesions in SLE.

In the present study, we evaluated the prevalence of 2dimensional and Doppler echocardiographic abnormalities and aPL [lupus anticoagulant (LA) and anticardiolipin antibodies (aCL)] in patients with SLE in Recife, Brazil, and analyzed the association between the presence of antibodies and cardiac lesions detected.

\section{Methods}

Seventy consecutive patients with SLE (67 women, 3 men) being treated either as inpatients or outpatients at the Hospital Agamenon Magalhães, the Hospital das ClínicasUFPE, the Hospital Oswaldo Cruz-UPE, and the Real Hospital Português, all general hospitals located in Recife, Brazil, were enrolled in this study. All patients fulfilled the 1982 re- 
vised criteria of the American College of Rheumatology ${ }^{14}$. Patients with a history of cancer, drug abuse, previous rheumatic fever, coagulation disturbances, or that were currently receiving anticoagulant drugs were excluded.

In addition, 42 female volunteers being treated as outpatients in the Gynecological Cancer Prevention Center of the Hospital das Clínicas-UFPE served as controls. All were premenopausal women with no clinical evidence of cardiac or systemic complaints, and no history of cancer, drug abuse, SLE, APL syndrome, or any immunological disturbances. A document of consent was signed by all patients and controls.

The tests for the lupus anticoagulant were carried out with the dilute Russell viper venom time (dRVVT) ${ }^{15}$. Blood was collected in one-tenth volumes of $3.8 \%$ trisodium citrate and was centrifuged at $1500 \mathrm{~g}$ for 30 minutes at room temperature to obtain platelet-poor plasma. All the samples were frozen in small aliquots at $-70^{\circ} \mathrm{C}$ before testing. The values considered normal for the test varied between 26.6 and 28.8 seconds and were obtained from a pool of plasma samples from normal patients, industrially fabricated (IL Test TM Plasma Control Normal, Instrumentation Laboratory Company, Lexington, MA/USA). Two reagents were utilized for gauging the dRVVT and to confirm the existence of phospholipid inhibitors, respectively: a reagent mixture containing Russell viper venom $(<8 \mathrm{mg} / \mathrm{kg})$, antiheparin agent (polybrene) and calcium (IL Test TM lupus anticoagulant Screen, Instrumentation Laboratory Company, Lexington, MA/USA), and another reagent mixture of identical content, except for an additional quantity of phospholipids (IL Test TMLA Confirm, Instrumentation Laboratory Company, Lexington, MA/USA).

The test was considered to indicate an alteration when the time for coagulation of the patient's plasma sample was at least $20 \%$ greater than the time obtained for the coagulation of the pool of plasma from normal patients (dR $V V T / d R V V T n$ ratio $\geq 1.2$ ). In the tests considered to show alteration, the presence of the lupus anticoagulant as a coagulation inhibitor and a cause of the increase in coagulation time was confirmed through the reagent with a higher concentration of phospholipids ${ }^{16-18}$. A ratio between the time using the low-phospholipid reagent and that using the high-phospholipid reagent equal to or greater than 1.2 was considered positive.

The aCL test was carried out on patients and controls as described previously ${ }^{19}$. Briefly, increasing dilutions of serum samples in a $10 \%$ solution of FCS/PBS were applied in duplicate to ELISA plates that had been previously sensitized with $25 \mathrm{mg} / \mathrm{mL}$ of cardiolipin in ethanol, treated with a $10 \%$ solution of skimmed milk, then incubated at $4^{\circ} \mathrm{C}$. After washing the plates with a $0.05 \%$ Tween $20 / \mathrm{PBS}$ solution, the anti-IgG, anti-IgM, or anti-IgA antibody, together with peroxidase or phosphatase alkaline diluted 1:5000, was added separately for 1 hour at ambient temperature. The reaction was revealed by the addition of ABTS substrate for peroxidase or pNNN for phosphatase alkaline as recommended by the manufacturer. The absorption reading was at $405 \mathrm{~nm}$.
The analysis of the mean optical density of each dilution of patient and control serum enabled the identification of the best dilution for detection of each immunoglobulin isotope, which was 1:128 for IgA and 1:256 for IgG and IgM. For each class of antibodies, samples with optical density values higher than the mean (by 5 standard deviations of 6 controls considered negative) were considered positive for the presence of the isotopes $\operatorname{IgG}, \operatorname{IgM}$, or $\operatorname{Ig} \mathrm{A}^{20}$.

Transthoracic Doppler echocardiographic examinations were carried out by a single examiner, usually within a period of up to 7 days following the interview and blood collection for antiphospholipid antibody tests. The equipment used for all Doppler echocardiographic examinations was an Interspect Apogee model with a $2.75 \mathrm{MHz}$ transducer, capable of performing unidimensional and bidimensional studies using continuous and pulsed Doppler. The echocardiographic measurements were made in accordance with the norms suggested by the American Echocardiographic Society ${ }^{21}$. The images for bidimensional studies were also obtained according to the usual standardization ${ }^{22}$. Doppler flow studies and bidimensional imaging were carried out simultaneously. The presence of pericardial effusion, global or segmental contractile deficit of the left ventricle (LV), diastolic dysfunction of the LV, left ventricular hypertrophy (LVH), pulmonary arterial hypertension, valvar thickening, and valvar regurgitation were registered. Mitral valve prolapse was not considered an alteration pertaining to the pathological spectrum in this study.

In bidimensional images, mild pericardial effusion was defined as the presence of isolated posterior effusion, moderate pericardial effusion as the presence of anterior and posterior effusion, and effusion of great magnitude as the presence of anterior and posterior effusion and abnormal excessive cardiac movements ("swimming heart").

The ejection fraction (EF) of the left ventricle (LV) was used as an indicator of the magnitude of the systolic dysfunction of the LV. Myocardial damage was defined as the presence of global contractile deficit of the $\mathrm{LV}(\mathrm{EF}<0.60)$, in the absence of clinical and/or electrocardiographic data of myocardial ischemic disease, or both, with mild systolic dysfunction reported when EF was $0.50-0.60$, moderate systolic dysfunction reported when EF was $0.40-0.49$, and systolic dysfunction of a great magnitude reported when EF $<0.40$.

Left ventricular hypertrophy was defined as a left ventricular wall thickness of more than $12 \mathrm{~mm}$ in diastole. Valvar thickness was measured from the parasternal long axis view and valvar thickening was reported if thickness was $3 \mathrm{~mm}$ or more.

Pulmonary artery systolic pressure (PASP) was calculated with the sum of the maximum pressure gradient in tricuspid insufficiency, shown by continuous Doppler, with an estimated right atrium pressure of $10 \mathrm{mmHg}$, which was considered equal to PSAP in the absence of right ventricle flow obstruction ${ }^{23}$. Patients that displayed a PSAP greater than $30 \mathrm{mmHg}$ were considered to be suffering from $\mathrm{PH}^{24-26}$. The mild form of PH was defined as the presence of PSAP 
31-50 $\mathrm{mmHg}$, the moderate form as the presence of PSAP 51$70 \mathrm{mmHg}$, and the severe form as the presence of PSAP $>70$ $\mathrm{mmHg}$.

The results were analyzed with Epi Info software, version 6 . The data obtained were expressed as mean + standard deviation. The Dopplerechocardiographic morphological findings were related to the presence or absence of antiphospholipid antibodies with the chi-square test. Variance analysis was used to estimate the difference between means. A value of $P$ lower than 0.05 was considered significant.

\section{Results}

In the group of lupus patients, the mean age was $33.5 \pm 10.6$ years (range of $13-65$ years), 3 patients being male and 67 female. The mean disease duration time was $5.8 \pm 5.4$ years (range of 1-32). Sixty-five patients (92.9\%) were using corticosteroids. The mean duration of corticosteroid use was $3.4 \pm 4.2$ years (range of 0.08 to 18 years). Fifteen patients (21.4\%) used cytotoxins in the 30-day period prior to interview. The control group, composed of 42 patients, had a mean age of $32.8 \pm 6.5$ years (range ef $19-44$ years), all being female. The clinical characteristics of patients with SLE are summarized in Table I.

Doppler echocardiographic abnormalities considered compatible with cardiopathy were registered in 38 of the 70 patients with SLE (54.3\%).

Pericardial effusion was observed in 8 of $70(11.4 \%)$ patients with SLE and in none of the controls $(P=0.02)$. Mild effusions were found in 5 of $8(62.5 \%)$ patients, 2 of $8(25 \%)$ having effusion considered moderate and 1 of $8(12.5 \%)$ effusion of great magnitude. No echocardiographic evidence

\begin{tabular}{|lcc|}
\hline \multicolumn{3}{|c|}{ Table I - Clinical characteristics of 70 patients with SLE * } \\
\hline Clinical data & Number of patients & Percentage \\
\hline Age (years) & $33.5 \pm 10.6$ &.. \\
Duration of disease (years) & $5.8 \pm 5.4$ &.. \\
Sex (female) & 67 & 95.7 \\
Cutaneous involvement & 54 & 77.1 \\
Oral ulcers & 21 & 30.0 \\
Articular involvement & 64 & 91.4 \\
Serositis & 28 & 40.0 \\
Systemic hypertension & 20 & 28.6 \\
Nephritis & 51 & 72.9 \\
Lesion of nervous system & 23 & 32.9 \\
Anemia & 36 & 51.4 \\
Leucopenia & 27 & 38.6 \\
Lymphopenia & 38 & 54.3 \\
Plaquetopenia & 11 & 15.7 \\
Antinuclear factor & 68 & 97.1 \\
Raynaud's phenomenon & 26 & 37.7 \\
Thrombotic events \# & 12 & 17.1 \\
Miscarriages & 17 & 25.0 \\
Use of corticosteroids & 65 & 92.9 \\
Use of cytotoxics & 15 & 21.4 \\
\hline * Present anytime during evolution of SLE; ${ }^{*}$ Myocardial & infarction, \\
cerebrovascular accident, deep venous thrombosis and/or arterial & & \\
occlusion of upper or lower limbs. & & \\
\hline
\end{tabular}

of tamponade or pericardial constriction was found in the patients studied.

Myocardial damage $(\mathrm{EF}<0.6)$ was present in 14 of 70 (20\%) patients with SLE and in none of the controls $(P=0.005)$. In the lupus patients with myocardial damage, anemia (serohemoglobin $\leq 10 \mathrm{mg} / 100 \mathrm{~mL}$ ) in $78.5 \%$ of cases $(P=0.04)$ and systemic arterial hypertension (blood pressure $>140 \times 90 \mathrm{mmHg})$ in $35.7 \%$ of patients $(P=0.51)$ were detected. Findings suggesting heart failure was detected in $2.86 \%$ of patients with SLE. Eight of the 70 lupus patients (11.4\%) were considered to have mild systolic dysfunction (EF 0.50$0.60)$, whilst 5 of $70(7.1 \%)$ displayed moderate systolic dysfunction $(\mathrm{EF}=0.40-0.49)$ and 2 of $70(2.9 \%)$ systolic dysfunction of a great magnitude $(\mathrm{EF}<0.40)$. The mean measurement of $E F$ of $L V$ of lupus patients was $0.66 \pm 0.09$, whilst the mean for control group patients was $0.69 \pm 0.03$ $(P=0.04)$. Segmental contractile deficit of $\mathrm{LV}$ was found in the echocardiogram to be present in 4 of $70(5.7 \%)$ patients with SLE and in none of the controls $(P=0.44)$.

LVH was present in 21 of $70(30 \%)$ patients and in none of the controls $(P<0.001)$. Twelve of the 21 lupic patients with LVH $(57.1 \%)$ displayed systemic hypertension ( $\mathrm{PR}=$ $3.33 ;$ CI95\% $=1.67$ to $6.66 ; P=0.001$ ).

$\mathrm{PH}$ was detected in the Doppler echocardiogram of 15 of $70(21.4 \%)$ patients with SLE and in 3 of $42(7.1 \%)$ control group individuals $(P=0.03)$. PSAP was used as an indicator of the magnitude of $\mathrm{PH}$. Twelve of the 15 patients with SLE and $\mathrm{PH}(80 \%$ ) displayed the mild form of PH (PSAP $31-50 \mathrm{~m}$ $\mathrm{mHg}$ ), 2 of 15 (13.3\%) the moderate form (PSAP $51-70 \mathrm{~m} \mathrm{~m}$ $\mathrm{Hg}$ ), and 1 of $15(6.6 \%)$ the severe form (PSAP> $70 \mathrm{mmHg}$ ). The 3 individuals of the control group with raised PSAP displayed forms of $\mathrm{PH}$ considered mild, with figures of 31 , 34 , and $37 \mathrm{mmHg}$. The mean measurements of PSAP in the group of patients with SLE was $40.5 \pm 14.1 \mathrm{mmHg}$, detecting a value of $30.8 \pm 5.1 \mathrm{mmHg}$ in the individuals of the control group $(P<0.001)$.

Valvar alterations not related to mitral valve prolapse were found in 5 of 70 patients $(7.1 \%)$ with SLE and in none of the controls $(P=0.07)$. Minimal aortic insufficiency was observed in 1 of 5 patients (20\%), in whom hypertension and LVH were detected. Mitral insufficiency (MI) was found in 4 of $5(80 \%)$ lupus patients, in all cases of a small degree. One of these cases of MI was associated with calcification of the mitral ring, 1 was associated with thickening of the mitral valve, and in 2 other cases the regurgitation was considered functional, associated with dilation of the left ventricle. MI of minimal degree was detected in 2 lupus patients with normal leaflets, and among the control group patients, 4 cases of mild MI in patients with normal mitral leaflets were also registered. These cases of minimal MI with normal mitral leaflets were not considered pathological ${ }^{4}$ and were not included in statistical analysis. None of the patients or controls displayed any clinical data that might indicate hemodynamic repercussion of valvar alterations detected on the Doppler echocardiogram.

Lupus anticoagulant was present in 7 of $70(10 \%)$ patients with SLE and in none of the controls $(P=0.03)$. The 
mean Russell time obtained on sampling for the lupus anticoagulant test in patients with SLE was $32.2 \pm 12$ seconds and in the controls was $25.6 \pm 2.1$ seconds $(P<0.001)$. The results of the sampling and confirmatory tests of lupus anticoagulant-positive patients with SLE are shown in Table II.

Among the lupus patients with cardiopathy, 4 of 38 $(10.5 \%)$ displayed a positive test for lupus anticoagulant $P=0.81)$. In the 3 lupus anticoagulant-positive lupus patients without cardiopathy, aPL syndrome findings were present, that is plaquetopenia, episodes of miscarriage, and evident livedo reticularis of the hands, respectively.

The lupus anticoagulant did not show itself to be significantly associated with the presence of pericardial effusion, myocardial dysfunction, myocardial ischemic disease, $\mathrm{LVH}$, $\mathrm{PH}$, or valvar disease. Similarly, no statistically significant association of the presence the lupus anticoagulant with plaquetopenia, history of miscarriage, or thrombotic events (cerebrovascular accident, deep venous thrombosis, peripheral arterial occlusion, or myocardial infarction at some time in clinical evolution of patients) was found.

The aCL IgG were present in 13 of $(18.6 \%)$ patients with SLE and in 4 of $42(9.5 \%)$ control group individuals $(P=0.30)$. In the group of lupus patients, aCL IgG were detected in 10 of $38(26.3 \%)$ patients with Doppler echocardiographic alterations, a trend towards statistical significance being observed $(\mathrm{PR}=1.57$; IC $95 \%=1.05$ to $2.33 ; P=0.06)$. Meanwhile, the aCL IgG were detected in 6 of $15(40 \%) \mathrm{PH}$ cases, with statistical significance $(\mathrm{PR}=2.92 ; \mathrm{CI} 95 \%=1.26$ to $6.77 ; P=0.02$ ). Positive results for aCL IgG, IgM, and IgA are shown in Table III.

Only 1 patient (1.4\%) positive for aCL IgA was observed among the lupus patients and 1 of $42(2.3 \%)$ among the controls $(P=0.71)$.

When aCL IgG, IgM, and IgA were evaluated as a whole, it was observed that aCL were detected in 31 of 70 (44.3\%) patients with SLE and in 14 of 42 (33.3\%) control group individuals $(P=0.34)$. The aCL were present in 17 of 38 $(44.7 \%)$ lupus patients with Doppler echocardiographic alterations and in 14 of $32(43.7 \%)$ of lupus patients without these alterations $(P=0.87)$.

\begin{tabular}{|lcccc|}
\hline $\begin{array}{l}\text { Table II - Values of dRVVT for seven } 7 \text { lupus anticoagulant-positive } \\
\text { lupus patients and their relationship both with dRVVT of the normal } \\
\text { pool and with the time found using the high-phospholipid reagent }\end{array}$ \\
\hline $\begin{array}{l}\text { dRVVT } \\
\text { (seconds) }\end{array}$ & DRVVT/ & DRVVT/ & Presence & Cardiopathy \\
85.9 & 2.98 & 2.27 & High & Mild PH \\
50.2 & 1.74 & 1.52 & Moderate & LV systolic dysfunction \\
45.7 & 1.59 & 1.54 & Moderate & LV dilation and LVH \\
71.1 & 2.47 & 1.97 & Moderate & Absent \\
68,9 & 2.51 & 1.65 & Moderate & Absent \\
63.4 & 2.31 & 1.84 & Moderate & Moderate PH \\
42.1 & 1.50 & 1.30 & Low & Absent \\
\hline \multicolumn{5}{|c}{} \\
$\begin{array}{l}\text { DRVVT- dilute Russell viper venom time; dRVVTn- dRVVT of the normal } \\
\text { pool; }\end{array}$ & dRVVTc=dRVVT with the use of high-phospholipid reagent \\
(confirmatory); PH- pulmonary arterial hypertension; LV- left ventricle; LVH- \\
left ventricular hypertrophy.
\end{tabular}

\begin{tabular}{|c|c|c|c|c|}
\hline \multicolumn{5}{|c|}{$\begin{array}{c}\text { Table III - Doppler echocardiographic and aPL syndrome findings in } \\
70 \text { patients with SLE. Comparison between positive results for aPL } \\
\text { and aCL IgG and IgM }\end{array}$} \\
\hline № patients & $\mathrm{aPL}(+)^{\#}$ & $\mathrm{aCL}(+)$ & ACL IgG (+) & $\operatorname{aCL} \operatorname{IgM}(+)$ \\
\hline Pericardiopathy & 3 & 3 & 0 & 3 \\
\hline LVH & 8 & 7 & 5 & 4 \\
\hline \multicolumn{5}{|l|}{ LV dysfunction } \\
\hline Global & 4 & 2 & 1 & 2 \\
\hline Segmental & 3 & 3 & 2 & 2 \\
\hline \multicolumn{5}{|l|}{ Valvopathy } \\
\hline MI & 2 & 2 & 2 & 1 \\
\hline AI & 1 & 1 & 1 & 0 \\
\hline $\begin{array}{ll}\mathrm{PH} & 15\end{array}$ & 10 & 9 & $6 *$ & 6 \\
\hline Thrombotic events 12 & $10 *$ & $9 *$ & 4 & 4 \\
\hline Miscarriages $\quad 17$ & 8 & 8 & 5 & 5 \\
\hline Plaquetopenia & 6 & 5 & 1 & 4 \\
\hline \multicolumn{5}{|c|}{$\begin{array}{l}\text { LVH- left ventricular hypertrophy; LV- left ventricle; MI- mitral } \\
\text { insufficiency; AI- aortic insufficiency; PH- pulmonary arterial hypertension; } \\
\text { \# Includes lupus anticoagulant-positive patients; } * \text { p }<0.05 \text {. }\end{array}$} \\
\hline
\end{tabular}

Patients with lupus anticoagulant and/or positive for aCL were considered positive for the presence of aPL. The frequency of aPL in patients with SLE was 35 of $70(50 \%)$ and 14 of $42(33.3 \%)$ in the controls $(P=0.12)$. The aPL were present in 19 of $38(50 \%)$ lupus patients with Doppler echocardiographic alterations and in 16 of $32(50 \%)$ lupus patients without these alterations. No statistically significant association was found between aPL evaluated as a whole and the Doppler echocardiographic alterations, whether evaluated together $(P=0.81)$ or separately.

With respect to thrombotic events, it was verified that aCL were present in 9 of 12 (75\%) lupus patients with current or previous thrombotic events, and in 22 of 58 (37.9\%) lupus patients without these alterations, with statistical significance $(\mathrm{PR}=3.77 ; \mathrm{CI} 95 \%=1.12$ to $12.77 ; P=0.04)$. In turn, aPL were present in 10 of $12(83.3 \%)$ lupus patients with thrombotic events and in 25 of $58(43.1 \%)$ lupus patients without these alterations, also with statistical significance ( $\mathrm{PR}=5$; CI 95\% $=1.18$ to $21.19 ; P=0.02$ ). No significant association was found between aCL or aPL and plaquetopenia or a history of miscarriage.

\section{Discussion}

Lupus anticoagulant was detected in 7 of $70(10 \%)$ patients, showing a frequency comparable to that of other reports in which similar methods were used ${ }^{27,28}$. In the present study, the presence of lupus anticoagulant did not show itself to be significantly associated with Doppler echocardiographic findings, whether evaluated together or separately. Among 7 lupus anticoagulant-positive lupus patients (10\%), 4 (57\%) demonstrated Doppler echocardiographic alterations, 2 of them $(28.5 \%)$ showing $\mathrm{PH}$ and another 2 $(28.5 \%)$ having myocardial damage.

The literature includes reports of occasional observations of lupus anticoagulant-positive lupus patients displaying mitral valvopathy and thrombosis in heart chambers ${ }^{29,30}$. An association between cardiac damage in SLE specifically 
with lupus anticoagulant has been researched by Jouhikainem et al ${ }^{31}$ who studied data on 74 patients with SLE following an evolution period averaging 22 years. The authors observed 5 cases ( $7 \%$ ) of valvar disease in the group of 37 lupus anticoagulant-positive patients and no cases in the same-sized group of lupus anticoagulant-negative lupus patients $(P=0.05)$. No indication of any statistically significant difference existed in relation to the number of cases of pericarditis, myocardial infarction, angina and heart failure, considering a tendency for lupus anticoagulant to be associated with cor pulmonale, a condition observed in 2 lupus anticoagulant-positive patients (3\%). Although the lupus patients in the present study had not been selected on the basis of being lupus anticoagulant-positive or not, 2 lupus anticoagulant-positive cases (285\%) with concurrent $\mathrm{PH}$ were also observed.

Among the patients demonstrating lupus anticoagulant and Doppler echocardiographic abnormalities, 2 also showed alterations potentially associated with aPL syndrome. One of them was suffering from acute arterial occlusion of the right upper member, and another related an isolated episode of recurrent miscarriage. Furthermore, livedo reticularis, plaquetopenia, and another report of isolated miscarriage were registered in the patients with lupus anticoagulant but without Doppler echocardiographic abnormalities. Although no statistically significant association for these findings was found, it should be considered that the relatively low prevalence of lupus anticoagulant among lupus patients results in only relatively large groups of lupus anticoagulant patients being able to consistently demonstrate significant associations of the latter with detection of aPL syndrome ${ }^{28}$. Nevertheless, thrombotic events significantly associated with lupus anticoagulant were observed in the groups of Petri et $\mathrm{al}^{27}$, in which lupus anticoagulant was detected in 4 of $60(6.7 \%)$ patients with SLE.

The association of aPL with cardiopathy in SLE has been most commonly reported in studies that include tests for lupus anticoagulant carried out in conjunction with research into aCL, or only the latter. The majority of significant associations found have been related to valvar dysfunctions ${ }^{2,3,5}$. In the present study, an association of aPL with valvar dysfunction was not found. Although the low frequency of valvar dysfunctions found here $(7.14 \%)$ should be considered, other authors did not detect an association between these findings and aPL ${ }^{10-12,32}$.

Li et al ${ }^{32}$ evaluated 50 Chinese patients with SLE and the association of their Doppler echocardiographic alterations with aPL, which were measured with ELISA for aCL IgG. The authors did not find a statistically significant association between the presence and gravity of valvar lesions and $\mathrm{aCL}$, and considered that racial differences might be implicated in the disparity between their results and those of other studies in which patients of Caucasian origin were involved. Although it is not possible to establish a perspective of racial homogeneity for the patients in the present study, who are from a country where racial mixing is a social reality, it is pertinent to consider that racial factors may have an influen- ce on the association of these valvopathies with aPL, as well as on the prevalence of valvar damage itself, in SLE ${ }^{33}$.

The frequency of cardiopathy in lupus patients in the present study (54.3\%) was similar to that found in other research $^{5,11,34-36}$, with the exception of valvar dysfunctions. We consider it possible that the low frequency $(7.1 \%)$ and the reduced magnitude of valvar alterations found in the present study are a consequence of an underestimation of the true frequency and magnitude of valvopathy in patients with SLE in the population evaluated, which presents a high prevalence of rheumatic cardiopathy. The possibility of lupus patients affected by Libman-Sacks endocarditis being confused with those with rheumatic disease or subacute bacterial endocarditis has already been pointed out by Gross ${ }^{37}$. In this way, patients previously diagnosed as suffering from chronic rheumatic valvar disease, and being in fact lupus patients with valvar disease, could have inadvertently been excluded from the evaluation. In another study carried out in Brazil, Macêdo ${ }^{38}$ observed a higher frequency of valvopathy in patients with SLE, but was unable to find a statistically significant difference between the frequencies of valvopathy in patients with SLE (28.3\%) and those of the control group (26\%), this being attributed to a possibly high prevalence of subclinical rheumatic cardiopathy in the study's target population.

It may also be supposed that the prolonged use of corticosteroids in $65(92.9 \%)$ of the lupus patients, 51 (72.8\%) of them having been using corticosteroids for 12 consecutive months or more, may have contributed to the reduced frequency of echocardiographic detection of Libman-Sacks endocarditis in this study. Through necropsy, Bulkley and Roberts ${ }^{39}$ observed a frequency of $18 \%$ of Libman-Sacks endocarditis in 36 patients with SLE using corticosteroids, verifying that endocardial lesions were considerably smaller in number and size than those found in necropsy of patients with SLE carried out before the advent of corticosteroid use.

In the present study, a tendency for aCL IgG to be associated with all Doppler echocardiographic alterations taken together $(P=0.06)$ was demonstrated. Nihoyannopoulos et $\mathrm{al}^{4}$ found an association between aPL and valvar dysfunctions in 93 patients with SLE, and also observed an association of aCL IgG and IgM with all the Doppler echocardiographic alterations taken together. The authors comment on the possibility of cardiac involvement in SLE being measured by aPL, possibly owing to the primary stimulation of the vascular endothelium. A direct lesion of the endothelium produced by aPL, leading to the activation of coagulation factors, consumption of platelets and the formation of thrombi, in conjunction with alterations in the interaction between platelets, circulating proteins, and endothelium in the cascade of coagulation, have been suggested as possible mechanisms for explaining Libman-Sacks endocarditis and other cardiac lesions in $\mathrm{SLE}^{40}$.

Meanwhile, Asherson and Cervera ${ }^{9}$ comment on the possibility that aPL may not be the direct cause of cardiac lesions, constituting simple epiphenomena accompanying 
other basic immunological disturbances. Furthermore, aPL could also be activated secondarily by exposed antigens in valve tissue previously damaged by other autoantibodies ${ }^{41}$.

In the present study, a significant association between $\mathrm{PH}$ and $\mathrm{aCL}$ IgG was also found. PH has been observed in Doppler echocardiograms and related to aPL in various studies ${ }^{6,25,42}$. The relation between aPL and PH does not appear to be absolute, and its pathogenic mechanisms are unclear ${ }^{9}$. The pathogenesis of PH in SLE has been related to the lesion of vascular endothelium by $\mathrm{aPL}^{43}$, and also to vasospasm and Raynaud's phenomenon rather than to aPL ${ }^{24}$. Nevertheless, even though the thromboembolic form of PH may be considered less frequent in SLE than the vasospastic form, in the latter aPL would also be concurrently observed ${ }^{43}$.

IgG class antibodies have been more frequently associated with thrombotic events than those of IgG and IgA classes ${ }^{44}$, it being possible that aCL IgM and IgA in the absence of IgG and/or lupus anticoagulant do not represent a risk of thrombosis ${ }^{42}$. Even though it should be considered that PH in SLE might not necessarily be of thromboembolic origin, the detection in this study of an association of $\mathrm{PH}$ with aCL IgG, but not with aCL IgM or IgA, is in agreement with the above-mentioned observations.

A limitation of this study could be related to the nonutilization of lupus activity indices for the evaluation of the patients, given that lupus activity has been related to higher frequencies of cardiopathic findings in some studies ${ }^{10,45,46}$. However, no association was found between the activity of the disease and a higher percentage of Doppler echocardiographic alterations in other groups of patients with SLE ${ }^{11,35}$. Another limitation could be linked to the fact that certain marginal forms of PH may be present without backflow through the tricuspid valve, preventing the evaluation of PASP by Doppler. Nevertheless, Murata et al ${ }^{23}$ believe that the absence of tricuspid regurgitation on Doppler implies normal pulmonary pressures.
At the moment, no clear explanation exists for the high percentage of aCL-positive control group patients (33.3\%) detected in this study. aPL would have a frequency of 2 to $10 \%$ of the general population ${ }^{47}$ and can be present in various other conditions, such as cancer, drug abuse, acquired immunodeficiency syndrome, and syphili ${ }^{48-50}$. In the present study, cancer or drug abuse were not present in the population studied, but syphilis and AIDS, whose identification usually requires serological tests, were not tested for. Future research, in which samples are obtained from a population evaluated for aPL, could also include these tests.

Harris ${ }^{19}$ and Harris et al ${ }^{51}$ observed that patients with syphilis may be positive on solid-phase immunological tests for the study of aCL, even though the antibodies of syphilitic patients may show a greater affinity for the antigenic complex (cardiolipin, cholesterol and lecithin) utilized in VDRL than for isolated cardiolipin used in solid-phase immunological tests.

In summing up, although no statistically significant association between aCL or aPL evaluated in conjunction with Doppler echocardiographic alterations taken together or separately was observed in the present study, aCL IgG showed a strong trend towards association with the Doppler echocardiographic alterations taken together $(\mathrm{PR}=1.57$; $\mathrm{CI} 95 \%=1.05$ to $2.33 ; P=0.06$ ), and were significantly associated with $\mathrm{PH}(\mathrm{PR}=2.92 ; \mathrm{CI} 95 \%=1.26$ to $6.77 ; P=0.02)$. The findings support the hypothesis of a primary pathogenic role of aCL IgG in relation to cardiac alterations in general and PH in particular, in SLE and in aPL syndrome.

\section{Acknowledgments}

We wish to acknowledge the help of Dr. Paula Loureiro, Dr. Paulo Miranda, Dr. Moacir Novaes, Dr. Emanuelli Tenório, Umbelina Raposo, Wayner de Souza, and Wlademir Gomes de Melo.

\section{References}

1. Chartash EK, Lans DM, Paget AS, et al. Aortic insufficiency and mitral regurgitation in patients with systemic lupus erythematosus and the antiphospholipid syndrome. Am J Med 1989; 86: 407-12.

2. Khamashta MA, Cervera R, Asherson RA, et al. Association of antibodies against phospholipids with heart valve disease in systemic lupus erythematosus. Lancet 1990; 335: 1541-4

3. Leung W-H, Wong K-L, Lau C-P, et al. Association between antiphospholipid antibodies and cardiac abnormalities in patients with systemic lupus erythematosus. Am J Med 1990; 89: 411-9.

4. Nihoyannopoulos P, Gomez PM, Joshi J, et al. Cardiac abnormalities in systemic lupus erythematosus: association with raised anticardiolipin antibodies. Circulation 1990; 89: 369-75.

5. Cervera R, Font J, Paré C, et al. Cardiac disease in systemic lupus erythematosus prospective study of 70 patients. Ann Rheum Dis 1992; 51: 156-9.

6. Asherson RA, Higenbottam TW, Dihn Xuan AT, et al. Pulmonary hypertension in a lupus clinic: experience with twenty-four patients. J Rheumatol 1990; 17: 1291-8.

7. Morton KE, Gavaghan TP, Krilis SA, et al. Coronary artery bypass graft failure an autoimune phenomenon? Lancet 1986; 1: 1353-7.

8. Hamstem A, Bkörkholm M, Norberg R, et al. Antibodies to cardiolipin in young survivors of myocardial infarction: an association with recurrent cardiovascular events. Lancet 1986, 1: 113-6.

9. Asherson RA, Cervera R. Antiphospholipids and the heart - lessons and pitfalls for the cardiologist. Circulation 1991; 84: 920-3.

10. Alves JA, Hydalgo L, Rolim LF, et al. Avaliação clínica e laboratorial da cardiopatia no lúpus eritematoso sistêmico. Arq Bras Cardiol 1997; 68: 79-83.

11. Ong ML, Veerapen K, Chambers JB, et al. Cardiac abnormalities in systemic lupus erythematosus: prevalence and relationship with disease activity. Int J Cardiol 1992; 34: 69-74.

12. Roldan CA, Shively BK, Lau CC, et al. Systemic lupus erythematosus valve disease by transesophageal echocardiography and the role of antiphospholipid antibodies. J Am Coll Cardiol 1992; 20: 1127-34.

13. Cervera R, Font J, Ingelmo M. Cardiac manifestations in the antiphospholipid syndrome. In: Asherson R, Cervera R, Piette J-C et al. (Eds). The Antiphospholipid Syndrome. Boca Raton: CRC Press, 1996: 151-60.

14. Tan EM, Cohen AS, Fries J, et al. Criteria for the diagnosis of systemic lupus erythematosus. Arthritis Rheum 1982; 25: 1271-7.

15. Thiagarajan $P$, Pengo V, Shapiro SS. The use of the dilute Russell viper venom time for the diagnosis of lupus anticoagulants. Blood 1986; 68: 869-74. 
16. Tripplet DA, Brandt J. Laboratory identification of the lupus anticoagulant. Br J Haematol 1989; 73: 139-42.

17. Rauch J, Tannembaum M, Janoff A. Distinguishing plasma lupus anticoagulants from anti-factor antibodies using hexagonal (II) phase phospholipids. Thromb Haemostas 1989; 62: 892-6.

18. Exner T, Tripplet DA, Taberner D, et al. Guidelines for testing and revised criteria for lupus anticoagulants. Thromb Haemostas 1991; 65: 320-2.

19. Harris EN. Antiphospholipid antibodies. Br J Haematol 1990; 74: 1-9.

20. Loizou JDS, McCrea AC, Rudge R, et al. Measurement of anti-cardiolipin antibodies by an enzyme-linked immunosorbent assay (ELISA): standardization and quantitation of results. Clin Exp Immunol 1985; 62: 738-45.

21. Sahn DJ, DeMaria A, Kisslo J, et al. Recommendations regarding quantitation in M-Mode echocardiography: results of a survey of echocardiographic measurements. Circulation 1978; 58: 1072-83.

22. Tajik A J, Seward JB, Hagler DJ, et al. Two-dimensional real-time ultrasonic imaging of the heart and great vessels - technique, image orientation, structure identification, and validation. Mayo Clin Proc 1978; 53: 271-303.

23. Murata I, Takenaka K, Toshinoya S, et al. Clinical evaluation of pulmonary hypertension in systemic sclerosis and related disorders: a Doppler echocardiographic study of 135 Japanese patients. Chest 1997; 111: 36-43.

24. Simonson JS, Schiller NB, Petri M, et al. Pulmonary hypertension in systemic lupus erythematosus. J Rheumatol 1989; 16: 918-25.

25. Sturfelt G, Eskilsson J, Nived O, et al. Cardiovascular disease in systemic lupus erythematosus - a study of 75 patients from a defined population. Medicine 1992; 71:216-23.

26. Winslon TM, Ossipov MA, Fazio GP, et al. Five-year follow-up study of the prevalence and progression of pulmonary hypertension in systemic lupus erythematosus. Am Heart J 1995; 129: 510-5.

27. Petri M, Rheinschmidt BAM, Whiting-O'Keefe Q, et al. The frequency of lupus anticoagulant in systemic lupus erythematosus. Ann Int Med 1987; 106: 524-31.

28. Feng C-S, Tsang SSF, Li EK. Evaluation of laboratory tests for lupus anticoagulant in a group of Chinese lupus patients. Pathology 1994; 26: 40-2.

29. Lubbe WL, Asherson RA. Intracardiac thrombus in systemic lupus erythematosus associated with lupus anticoagulant. Arthritis Rheum 1988; 31: 1453-4.

30. Ford PM, Ford SE, Lillicrap DP. Association of lupus anticoagulant with severe valvular heart disease in systemic lupus erythematosus. J Rheumatol 1988; 15 597-600.

31. Jouhikainem T, Pohjola-Sintonen S, Stephansson E. Lupus anticoagulant and cardiac manifestations in systemic lupus erythematosus. Lupus 1994; 3: 167-72.

32. Li E, Crozier IG, Milne MJ, et al. Lack of correlation between anticardiolipin antibodies and heart valve disease in Chinese patients with systemic lupus erythematosus. Lancet 1990; 336: 504-5.

33. Crozier I, Li E, Milne M, et al. Valvular disease in systemic lupus erythematosus. N Engl J Med 1989; 320: 739-40.

34. Leung W-H, Wong K-L, Lau C-P, et al. Cardiac abnormalities in systemic lupus erythematosus: a prospective M-Mode, cross-sectional and Doppler echocardiographic study. Int J Cardiol 1990; 27: 367-75.

35. Roldan CA, Shively BK, Crawford MH. An echocardiographic study of valvula heart disease associated with systemic lupus erythematosus. N Engl J Med 1996; 335: 1424-30

36. Galve E, Candell-Riera J, Pigrau C, et al. Prevalence, morphologic types, and evolution of cardiac valvular disease in systemic lupus erythematosus. N Engl J Med 1988; 319: 817-23.

37. Gross L. The cardiac lesions in Libman-Sacks disease - with a consideration of its relationship to acute diffuse lupus erythematosus. Am J Pathol 1940; 16 : 375-407.

38. Macêdo TB. Avaliação ecocardiográfica no lúpus eritematoso sistêmico: um estudo transversal de 53 pacientes (dissertation). Recife: Universidade Federal de Pernambuco, 1998: $100 \mathrm{p}$.

39. Bulkley HB, Roberts WC. The heart in systemic lupus erythematosus and the changes induced in it by corticosteroid therapy - a study of 36 necropsy patients. Am J Med 1975: 58: 243-64.

40. O'Rourke RA. Antiphospholipid antibodies: a marker of lupus carditis? Circulation 1990; 82: 636-8.

41. Hojnik M, George J, Ziporen L, et al. Heart valve involvement (Libman-Sacks endocarditis) in the antiphospholipid syndrome. Circulation 1996; 93: 1579-87.

42. Miyata M, Suzuki K, Sakuma F, et al. Anticardiolipin antibodies are associated with pulmonary hypertension in patients with mixed conective tissue disease or systemic lupus erythematosus. Int Arch Allergy Immunol 1993; 100: 351-4.

43. Asherson RA, Cervera R. Antiphospholipid antibodies and the lung. J Rheumatol 1995; 22: 62-6.

44. Petri M. Pathogenesis and treatment of the antiphospholipid antibody syndrome Med Clin North Am 1997; 81: 151-77.

45. Leung W-H, Wong K-L, Lau C-P, et al. Doppler echocardiographic evaluation of left ventricular diastolic function in patients with systemic lupus erythematosus. Am Heart J 1990; 120: 82-7.

46. Castier MB, Meneses ME, Albuquerque EM, et al. O envolvimento cardíaco no lúpus eritematoso sistêmico: uma avaliação ecocardiográfica. Arq Bras Card 1994; 62: 407-12.

47. Lockshin MD. Which patients with antiphospholipid antibody should be treated and how? Rheum Dis Clin North Am 1993; 19: 235-47.

48. Bick RL. The antiphospholipid-thrombosis syndromes: fact, fiction, confusion and controversy. Am J Clin Pathol 1993; 100: 477-80.

49. Bick RL, Kaplan H. Syndromes of thrombosis and hypercoagulability - congenital and acquired causes of thrombosis. Med Clin North Am 1998; 82: 409-58.

50. Harris EN. Antiphospholipid syndrome. In: Klippel JH, Dieppe PA. (Eds). Rheumatology (2nd ed.). London: Mosby, 1998; sec.7: 35.1-35.7.

51. Harris EN, Gharavi AE, Wasley GD, et al. Use of an enzyme-linked immunosorbent assay and of inhibition studies to distinguish between antibodies to cardiolipin from patients with syphilis or autoimmune disorders. J Infectious Dis 1988; 157: 23-31. 